\title{
MAGNETIC PROPERTIES OF CONDUCTING POLYMERS
}

\author{
O.I. Aksimentyeva, V.T. Artym, O.I. Melnik and T.A. Plusnina \\ Lviv State Ivan Franko University, Ukraine
}

\begin{abstract}
Magnetic susceptibility, electroconductivity and structure of polymer conducting layers of polyaniline and polyaminophenoles were studied in a wide temperature range. It was found that magnetic susceptibility depends on the doping level of polymer and the nature of doping agents. The obtained result suggested the existence of metallic states in highly doped conducting polymers.
\end{abstract}

PACS numbers: 81.20.Sh, 61.41.+e, 75.50.-y

\section{Introduction}

Conducting polymers on the basis of aromatic compounds have attracted great attention because of their electrical properties and high thermostability. The investigation of conducting mechanism in polymeric conductors is an actual problem for the real technology application. Basic structural requirement for an appearance of metallic state of the polymer is that the chain contains a repetition unit with mobile carrier and presence of effective electron transfer (exchange) interaction between these unit groups [1, 2].

To define the nature of free carriers in polyaromatic systems the temperature dependencies of magnetic susceptibility, electric conductivity in connection with polymer structure must be studied.

\section{Experimental}

Polyaniline (PAN), polymetaaminophenole (PMAP), polyortoaminophenole (POAP) were obtained as electrodeposited films on the $\mathrm{Pt}$, graphite surfaces at the current density $0.1-5.0 \mathrm{~mA} / \mathrm{sm}^{2}$, and synthesized by the interaction of aniline, $o-$, $m$-aminophenoles in aqueous sulfuric acid solution with ammonium persulfate at monomer-oxidant relationship 1:1. To obtain the basic form of PAN the precipitate was washed with $5 \% \mathrm{NH}_{4} \mathrm{OH}$, distilled water, acetone and dried in vacuum. Doping procedure includes the treatment of $\mathrm{PAN}$-base with $1 \mathrm{~N}$ aqueous $\mathrm{HCl}, \mathrm{HFB}_{4}$, $\mathrm{HClO}_{4}$ and saturated $\mathrm{I}_{2}$ in chloroform solution. All the materials were identified by IR, UV spectroscopy and elemental analysis. 
The X-ray diffraction analysis was performed on the DRON-2 diffractometer, where the Fe $K$ radiation was used. Electron-microscopic analysis was performed on the UEMB-100 and REM-200 instruments. For EPR spectroscopy the radiospectrometer AE 4700 was used. Magnetic susceptibility was studied with magnetic microbalance in the $77-295 \mathrm{~K}$ temperature range.

\section{Result and discussion}

We studied the molecular and submolecular structure of polymer layers of PAN, POAP, PMAP polymerized on electrodes in aqueous acid solution and chemically synthesized [3]. According to the X-ray researching the diffraction patterns of the conducting polyaromatic polymers show a maximum, which contains reflections in its substructure with information about ordering of the polymers. This makes it possible to suppose that the structure of these materials is influenced by the existence of both amorphous and crystalline phases. Indexes of crystallinity calculated by means of the Bochard method is in the range $25-71 \%$ (see Table).

TABLE

Structure of polymer conductors.

\begin{tabular}{|c|c|c|c|}
\hline Polymer & Molecular structure & $\begin{array}{l}\text { X-ray ref- } \\
\text { lection } 2 \Theta\end{array}$ & $\begin{array}{l}\text { Index of cry- } \\
\text { stallinity [\%] }\end{array}$ \\
\hline $\mathrm{PAN}_{\text {base }}$ & & $\begin{array}{l}25.0 ; 26.5 ; \\
31.4\end{array}$ & 25 \\
\hline PAN $_{\text {doped }}$ & & $\begin{array}{l}18.7 ; 22.35 \\
25.6 ; 32.25\end{array}$ & 41 \\
\hline POAP & $\mathbf{H}$ & $\begin{array}{l}15.5 ; 16.7 ; \\
19.6 ; 26.0 ; \\
31.6 ; 35.2 ; \\
40.2\end{array}$ & 65 \\
\hline PMAP & & $\begin{array}{l}48.0 ; 43.5 \\
34.5 ; 32.5 \\
21.5\end{array}$ & 23 \\
\hline
\end{tabular}

According to the electron microscopy and electronography the PAN-films (600-800 $\AA$ ) thickness consists of inhomogeneities by island nature (domains) on the 1-10 $\mu \mathrm{m}$ distance and the 0.1-3.0 $\mu \mathrm{m}$ diameter. The POAP films have the 
fibre structure with linear stretched fibre. X-ray diffraction patterns of polymer films obtained on graphite surface show that macrochain molecules in the interface have a coplanar orientation with the basic plane of substrate.

Experiments, involving electron paramagnetic resonance for chemically synthesized PAN, POMP and PMAP, have shown that signal intensivity for PAN is more than two orders higher in comparison with other polyaromatic conductors.

The centres of EPR signal for PAN, POAP and PMAP are at $3371-3372 \mathrm{~Hz}$, but the signal half-width is drastically different: for PAN a narrow signal is observed, while for POAP, PMAP its value increases 4-7 times. The loss of intensivity and boadering of EPR signals of polyaminophenoles correlates with these lower electrical conductivities in comparison with PAN.

On the basis of temperature dependencies of the aromatic polymers volume resistance it was established that conductivity of POAP, PMAP, undoped PAN and doped with iodine and sulfuric acid PAN is characterized by semiconductor behaviour with charge-transport activation energy $0.11-0.38 \mathrm{eV}$ (see Fig. 1). PAN highly doped with $\mathrm{HCl}$ demonstrates the independence of the resistance in

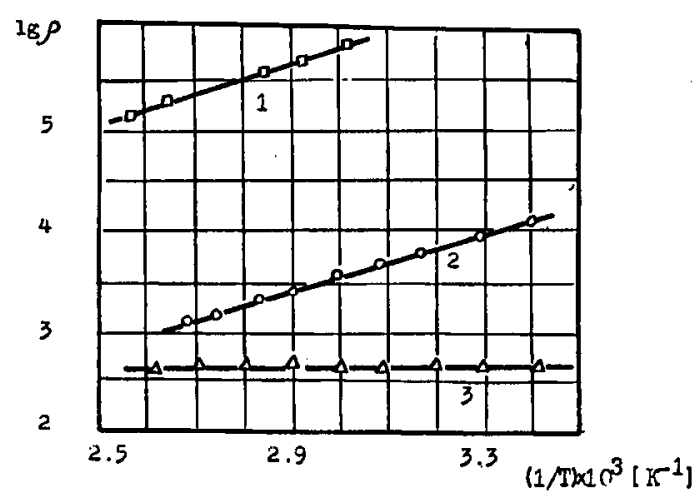

Fig. 1. Temperature dependence of resistance for the PAN doped by $\mathrm{Y}_{2}(1), \mathrm{H}_{2} \mathrm{SO}_{4}(2)$, $\mathrm{HCl}(3)$.

$278-403 \mathrm{~K}$ range, which suggests the existence of metallic-type conductivity in this material.

We have studied the temperature dependencies of magnetic susceptibility of PAN, doped by different dopants, in the temperature interval 77-297 K and the effect of the magnetic fields on magnetic susceptibility changing. The obtained results are shown in Fig. 2.

As follows from the obtained data, for the HCl-doped PAN the Curie and Pauli spin paramagnetism in the temperature intervals $80-180 \mathrm{~K}$ and $200-300 \mathrm{~K}$ are observed. It was noted that spin paramagnetism is due to the electron conductivity and for the first time has been observed by MacDiarmid and Epstein [4] in chemically synthesized PAN. The temperature independence Pauli term is $\chi=1.6 \times 10^{-4} \mathrm{sm}^{3} /$ two aniline groups for HCl-doped PAN $\left(7 \times 10^{-5}\right.$ e.m.u./two aniline groups for the $\mathrm{HBF}_{4}$-doped), according to the result obtained by Tinaka 


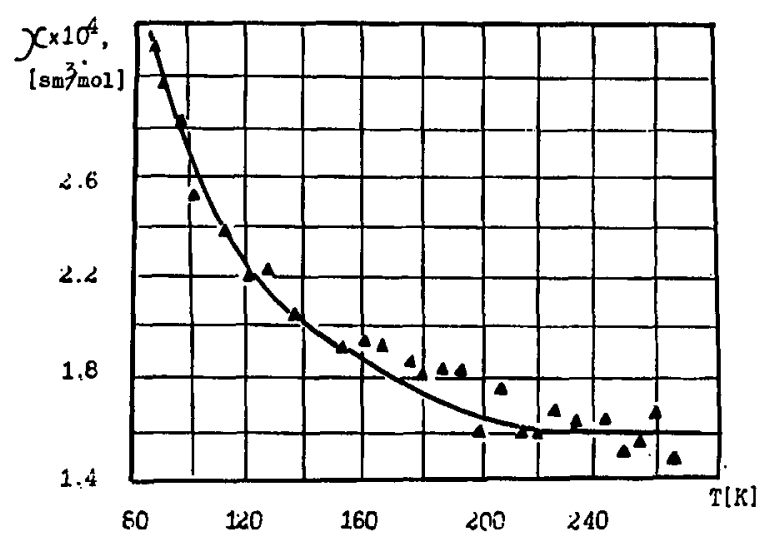

Fig. 2. Temperature dependence of magnetic susceptibility of PAN doped by HCl.

et al. [5]. This observation as well as resistance temperature independencies point out the metallic character of the polymer.

It was found that magnetic properties of doped polyaniline depend on doping agents and on the degree of oxiding; for $\mathrm{H}_{2} \mathrm{SO}_{4}$-doped PAN the diamagnetic effect took place in the range of temperature $80-290 \mathrm{~K}$ which is in the boundary interval with results obtained by Langer [6] (115-415 K).

\section{Conclusion}

The defining of magnetic properties of conducting polymer, in particular temperature dependencies of the magnetic susceptibility give information for the understanding of conducting mechanism in polyaromatic systems. Metallic character of conductivity is possible in highly acid-doped polyaniline by the electron delocalization along polymer chain and charge transfer among coplanar disposed aromatic rings.

\section{References}

[1] E.M. Genies, A. Boyle, M. Lapkowski, C. Tsintavis, Synth. Met. 36, 139 (1990).

[2] E.P. Kovalchuk, O.I. Aksimentyeva, A.P. Tomilov, Electrosynthesis of Polymers on the Surfaces of Metals, Khimiya, Moscow 1991, p. 222.

[3] V.T. Artym, O.I. Aksimentyeva, I.I. Artym, T.A. Plusnina, O.Y. Melnik, in: Condensed Matter: Science and Industry, Ukrainian-French Symposium, Lviv 1993, p. 88.

[4] J.M. Ginger, A.F. Richer. A.G. MacDiarmid, A.J. Epstein, Solid State Commun. 63, 97 (1987).

[5] J. Tinaka, N. Mashita, K. Mizoguchi, K. Kume, Synth. Met. 29, E175 (1989).

[6] J.J. Langer, J. Mol. Electron. 5, 103 (1989). 